\title{
Pathology of Sleep, Hormones and Depression
}

Authors

Affiliations
A. Steiger ${ }^{1}$, M. Dresler ${ }^{1}$, M. Kluge ${ }^{1,2}$, P. Schüssler ${ }^{1}$

${ }^{1}$ Department of Psychiatry, Max Planck Institute of Psychiatry, Munich, Germany

${ }^{2}$ Department of Psychiatry, University of Leipzig, Leipzig, Germany

\author{
Key words \\ - depression \\ - sleep \\ peptides \\ - memory \\ motoric learning
}

Bibliography

DOI http://dx.doi.org/

10.1055/s-0033-1337921

Pharmacopsychiatry 2013;

46 (Suppl. 1): S30-\$35

(c) Georg Thieme Verlag KG

Stuttgart · New York

ISSN 0176-3679

Correspondence

\section{Axel Steiger, MD}

Max Planck Institute of

Psychiatry

Kraepelinstraße 10

80804 Munich

Germany

steiger@mpipsykl.mpg.de

\section{Abstract}

$\checkmark$

In patients with depression, characteristic changes of sleep electroencephalogram and nocturnal hormone secretion occur including rapid eye movement (REM) sleep disinhibition, reduced non-REM sleep and impaired sleep continuity. Neuropeptides are common regulators of the sleep electroencephalogram (EEG) and nocturnal hormone secretion and changes in their activity appear to contribute to the aberrances of sleep in affective disorders. A reciprocal interaction of the sleep-promoting growth hormone-releasing hormone (GHRH) and corti-

\section{Introduction}

$\nabla$

Disturbed sleep and impaired cognition are frequent symptoms in patients with depression. Objective assessment of their sleep by polysomnography (sleep electroencephalogram [EEG]) reveals characteristic changes. Furthermore nocturnal hormone secretion shows aberrances in affective disorders. Here we review studies on the interaction between depression, sleep, memory and endocrine activity focusing particularly on work of our laboratory.

Simultaneous investigation of sleep EEG and nocturnal hormone secretion shows that in young healthy subjects during the first half of the night slow-wave sleep (SWS) and the major peak of growth hormone $(\mathrm{GH})$ secretion during $24 \mathrm{~h}$ preponderate, whereas the second half of the night is dominated by rapid eye movement (REM) sleep and the secretion of the hormones of the hypothalamic-pituitary-adrenocortical (HPA) system corticotrophin (ACTH) and cortisol [1]. During normal ageing and during an episode of depression changes of sleep-endocrine activity occur. Well documented sleep-EEG changes in depression include disinhibition of rapid eye cotrophin-releasing hormone (CRH), which promotes wakefulness and REM sleep, plays a key role in sleep regulation, at least in male subjects. Also galanin and ghrelin promote sleep in men. Neuropeptide $Y$ is involved in the timing of sleep onset. The effects of peptides of sleep are influenced by the time of administration, age, gender and depression. In healthy subjects and in remitted depressed patients motoric memory learning is consolidated during sleep. This effect is absent in depressed patients who are at least 30 years old, and is probably related to elevated glucocorticoid levels.

movement (REM) sleep (shortened REM latency, prolonged first REM period, elevated REM density, a measure of the amount of rapid eye movements during REM sleep), impaired non-REM sleep (reduced time spent in sleep stage 2 and SWS) and changes of sleep continuity (prolonged sleep latency, frequent nocturnal awakenings and early morning awakening) [2]. Similarly during aging more shallow sleep develops [3]. ACTH and cortisol secretion are elevated in depressed patients. The amplitude of the nocturnal course of these hormones is dampened in the elderly resulting in an elevated nadir. $\mathrm{GH}$ secretion is blunted during ageing and in a depressive episode as well [1]. The sleep-endocrine pattern in young healthy subjects and its changes related to depression and ageing suggest that there exist endogenous factors which are common regulators of sleep EEG and hormones, linking, for example, SWS and GH or HPA activity and REM sleep, respectively. 
Table 1 Effects of neuropeptides on human sleep.

\begin{tabular}{|c|c|c|c|c|c|c|c|c|c|}
\hline \multirow[t]{2}{*}{ Peptide } & \multirow[t]{2}{*}{ Sex } & \multirow[t]{2}{*}{ Sample } & \multirow[t]{2}{*}{ Time } & \multicolumn{6}{|c|}{ Effects on } \\
\hline & & & & wake & NonREM & REM & other & GH & Cortisol \\
\hline GHRH & $\mathrm{m}$ & h vol & so & $\downarrow$ & $\uparrow$ & $\div$ & & $\uparrow$ & $\downarrow$ \\
\hline GHRH & $\mathrm{m}$ & y h vol & morn. & $\div$ & $\div$ & $\div$ & & $(\uparrow)$ & $\div$ \\
\hline GHRH & $\mathrm{f} / \mathrm{m}$ & elder & so & $(\downarrow)$ & $(\uparrow)$ & $\div$ & & $(\uparrow)$ & $\div$ \\
\hline GHRH & $f$ & h vol & so & $\uparrow$ & $\downarrow$ & $\div$ & & $\uparrow$ & $\div / \uparrow$ \\
\hline GHRH & $\mathrm{m}$ & dep pat & so & $\downarrow$ & $\uparrow$ & $\div$ & & $\uparrow$ & $\downarrow$ \\
\hline GHRH & $f$ & dep pat & so & $\uparrow$ & $\downarrow$ & $\div$ & & $\uparrow$ & $\uparrow$ \\
\hline ghrelin & $\mathrm{m}$ & y h vol & so & $\div$ & $\uparrow$ & $(\downarrow)$ & & $\uparrow$ & $\uparrow$ \\
\hline ghrelin & $\mathrm{m}$ & y h vol & morn. & $\div$ & $\div$ & $\div$ & & $\uparrow$ & $\uparrow$ \\
\hline ghrelin & $\mathrm{m}$ & elder & so & $\div$ & $\uparrow$ & $\downarrow$ & & $\uparrow$ & $\uparrow$ \\
\hline ghrelin & $f$ & elder & so & $\div$ & $\div$ & $\div$ & & $\uparrow$ & $\uparrow$ \\
\hline ghrelin & $\mathrm{m}$ & dep pat & so & $\downarrow$ & $\uparrow$ & $\div$ & & $\uparrow$ & $\uparrow$ \\
\hline ghrelin & $f$ & dep pat & so & $\uparrow$ & $\div$ & $\downarrow$ & & $\uparrow$ & $\uparrow$ \\
\hline somatostatin & $\mathrm{m}$ & y h vol & so & $\div$ & $\div$ & $\div$ & & & \\
\hline somatostatin & $\mathrm{f} / \mathrm{m}$ & elder & so & $\uparrow$ & $\downarrow$ & $\downarrow$ & & & \\
\hline octreotide & $\mathrm{m}$ & y h vol & so & $\uparrow$ & $\downarrow$ & $\downarrow$ & & & \\
\hline $\mathrm{CRH}$ & $\mathrm{m}$ & y h vol & so & $\div$ & $\downarrow$ & $\downarrow$ & & $\downarrow$ & $\uparrow$ \\
\hline $\mathrm{CRH}$ & $f$ & y h vol & so & $\uparrow$ & $\downarrow$ & $\uparrow$ & & $\div$ & $\uparrow$ \\
\hline АCTH(4-9) & $\mathrm{m}$ & y h vol & so & $(\uparrow)$ & $(\downarrow)$ & & & $\div$ & $\div$ \\
\hline NPY & $\mathrm{m}$ & y h vol & so & $\div$ & $\uparrow$ & $\div$ & slat $\downarrow$, SPT $\uparrow$ & $\div$ & $\downarrow$ \\
\hline NPY & $\mathrm{f} / \mathrm{m}$ & h vol & so & $\div$ & $\div$ & $\div$ & slat $\downarrow$ & $\div$ & $\div$ \\
\hline NPY & $\mathrm{f} / \mathrm{m}$ & dep pat & so & $\div$ & $\div$ & $\div$ & slat $\downarrow$ & & \\
\hline galanin & $\mathrm{m}$ & y h vol & so & $\div$ & $\uparrow$ & $\div$ & & $\div$ & $\div$ \\
\hline galanin & $\mathrm{f} / \mathrm{m}$ & dep pat & day & $\div$ & $\div$ & $\div$ & REMlat $\downarrow$ & & \\
\hline
\end{tabular}

\section{Effects of Neuropeptides on Sleep}

Indeed preclinical studies have shown that the releasing hormones GH-releasing hormone (GHRH) and corticotrophinreleasing hormone $(\mathrm{CRH})$, besides their endocrine actions, regulate sleep. After central administration of GHRH to rats SWS increases, whereas CRH impairs sleep [4]. In mice overexpressing CRH in the central nervous system (CNS) REM sleep is elevated in comparison to the wild type [5]. We tested whether similar effects are found after pulsatile intravenous (i.v.) administration of these peptides to humans. Indeed in young male healthy volunteers after GHRH, SWS and GH increased and cortisol was blunted [6]. In contrast after CRH, SWS decreased during the second half of the night, the GH peak was blunted and cortisol increased during the first half of the night [7]. These findings (see also $\odot$ Table 1 ) confirm the view that a reciprocal interaction of GHRH and CRH plays a key role in sleep-endocrine regulation [8]. It is unlikely that the changes of sleep EEG after GHRH and CRH are mediated by the elevated secretion of GH or cortisol, respectively. Other studies showed that SWS decreases after acute administration of GH to humans [9] and laboratory animals [10], whereas SWS and GH are elevated after cortisol administration to healthy volunteers [11]. These changes can be explained by feed-back inhibition of the related releasing hormones GHRH and CRH. Therefore we suggest that peptides act directly in the brain and affect there sleep EEG. This view is supported by the observation that sleep EEG is influenced by the ACTH (4-9) analogue ebiratide. In young male volunteers after ebiratide a set of sleep-EEG changes occurred mirroring cerebral activation. In detail SWS decreased and sleep latency and wakefulness increased during the first third of the night. The plasma concentrations of cortisol and GH remained unchanged, however [12]. Similarly SWS increased in healthy male subjects after the neuropeptide galanin in absence of changes of GH and cortisol secretion [13]. The latter observation is in line with Saper's hypothesis that a cluster of neurons containing galanin and GABA in the ventrolateral preoptic area participates in the promotion of non-REM sleep [14]. The findings after ebiratide and after galanin administration demonstrate that peptides are able to modulate sleep EEG independently from peripheral hormones. Obviously the blood-brain interface is not an obstacle for these effects. Repetetive pulsatile administration of peptides appears to be a crucial methodological issue since after nocturnal infusions and single i.v. injections of GHRH sleep remained unchanged [15].

The effects of peptides on sleep EEG and hormone secretion are influenced by time of administration, age, sex and depression. The same dosage of GHRH which enhanced SWS after administration around sleep onset did not prompt major sleep-EEG changes when given during the morning hours, an interval with high endogenous HPA activity [16]. The response of GH after GHRH given at daytime is reduced in elderly men compared to young men [17]. Similarly GHRH administration to healthy women and men who were 60 years of age and older prompted only weak sleep promoting effects. In this sample the number of nocturnal awakenings decreased and the time spent in nonREM sleep during the first sleep cycle was prolonged [18]. A sexual dimorphism in the effects of GHRH on sleep-endocrine activity was found in a large sample of drug-free female and male patients with depression of a wide age range and in matched healthy controls. In male patients and control subjects a sleep promoting effect was confirmed as the amount of 
non-REM sleep increased and the time spent awake during the sleeping period decreased. Similar to our previous study HPA hormones were blunted as ACTH declined during the first half of the night and cortisol decreased during the second half of the night after this peptide $[19,20]$. In depressed and healthy women, however, opposite effects were found. Sleep was impaired as intermittent wakefulness increased and non-REM sleep decreased. ACTH and cortisol were elevated in female patients and control subjects $[19,20]$. This sexual dimorphism was confirmed in a study in young healthy female volunteers with injection of 2 dosages of GHRH or placebo during the same interval of the menstrual cycle. Again sleep impairing effects of GHRH were found in women, whereas cortisol levels did not differ between GHRH and placebo conditions [21].

Somatostatin is the counterpart of GHRH in the regulation of GH release. We tested whether similarly its action on sleep is opposite to that of GHRH. In young healthy men sleep remained unchanged after pulsatile i.v. somatostatin administration [6]. The same dosage of somatostatin which did not affect sleep in young healthy men impaired sleep in healthy seniors [22]. Obviously the sleep promoting effect of GHRH declines during ageing whereas the sleep impairing action of somatostatin increases. After subcutaneous administration of the highly potent somatostatin analogue octreotide, sleep was impaired in young healthy men [23]. These data suggest that somatostatin participates not only in the regulation of $\mathrm{GH}$ secretion but also in sleep regulation in an opposite fashion to GHRH.

The effects of CRH were more distinct in young healthy women than in young healthy men. In women CRH prompted a decrease of stage 3, one component of SWS, an increase of intermittent wakefulness and an increase of the time spent in REM sleep during the first third of the night, all changes resembling sleep-EEG alterations in depressed patients [24]. Vice versa during a clinical trial with the CRH-1-receptor antagonist R 121919 after 4 weeks the amount of SWS increased, the number of awakenings and REM density decreased [25]. These findings suggest that $\mathrm{CRH}$-1-receptor antagonism helps to normalize impaired sleep in depressed patients. Furthermore, the view is corroborated that CRH overactivity contributes to the sleep-EEG changes in depression.

Neuropeptide Y (NPY) is thought to be an endogenous CRH antagonist. In a preclinical study $\mathrm{CRH}$ prolonged sleep onset latency and decreased sleeping time in rats. These changes were counteracted dose-dependently by NPY [26]. In another study benzodiazepine-like EEG changes after NPY were described in rats [27]. Similarly after repetitive i.v. administration of NPY to young healthy men benzodiazepine-like changes of sleep EEG were found, including shortened sleep latency and increases of sleep period time and the time spent in sleep stage 2 . Nocturnal ACTH and cortisol levels decreased after NPY in this sample. These findings are compatible with a CRH antagonistic and a benzodiazepine-like action as well. In another study we investigated the sleep-endocrine effects of NPY in drug-free patients of both sexes and in matched healthy controls. These samples were older than the young volunteers in our previous study. Again sleep latency was prolonged after NPY, but other sleep-EEG variables and ACTH and cortisol secretion remained unchanged. Probably timing of sleep onset is a major effect of NPY [28].

Beside GHRH and somatostatin, ghrelin is the third peptide involved in GH secretion. Ghrelin binds to the $\mathrm{GH}$-secretagogue receptor and stimulates in addition to $\mathrm{GHRH}$ the secretion of $\mathrm{GH}$. Furthermore, it is involved in the energy balance as the most potent endogenous stimulus for food intake and body weight [29]. After repetitive i.v. injections of ghrelin to healthy young volunteers SWS and correspondingly EEG delta waves increased [30]. These effects resemble those of GHRH in young men. The pattern of endocrine changes after ghrelin differs, however, from the effects of GHRH in young male subjects. After ghrelin, $\mathrm{GH}$ and cortisol increased [31], whereas after GHRH GH increased, too, but cortisol decreased [6]. In a series of studies the influence of time of administration, age, sex and depression on the effects of ghrelin on sleep-endocrine activity were investigated. Similar to GHRH ghrelin did not affect sleep EEG after administration to healthy young men during the early morning hours [31]. A sleep-promoting effect of ghrelin was preserved in elderly healthy men as non-REM sleep increased [32]. In young [33] and in postmenopausal [32] healthy women, however, sleep EEG remained unchanged after ghrelin. Endocrine effects were similar in both sexes with increases of GH and cortisol. In male drugfree patients with depression again a sleep-promoting effect of ghrelin occurred. In contrast in female patients REM sleep decreased, whereas non-REM sleep remained unchanged [34]. The interactions between ghrelin, GHRH and CRH in sleep regulation and the secretion of cortisol and GH were studied in male healthy volunteers who underwent 4 protocols receiving placebo (A), ghrelin (B), ghrelin and GHRH (C), or ghrelin and CRH (D) in pulsatile fashion in a randomized cross-over study [35]. Non-REM sleep increased in all verum conditions compared to placebo. REM sleep was decreased in conditions B, C and D compared to placebo. After the combination of $\mathrm{CRH}$ and ghrelin the time spent awake decreased and sleep efficiency increased; furthermore, REM latency decreased compared to the other conditions. CRH enhanced the ghrelin-induced cortisol concentration but had no relevant effect on GH. In turn, after GHRH the ghrelin-induced GH concentration was elevated whereas cortisol secretion remained unchanged. In all, ghrelin exhibited distinct sleep effects, which tended to be enhanced by both GHRH and CRH. CRH exerted sleep-improving and REM permissive effects when co-administered with ghrelin.

As mentioned before galanin stimulates sleep in healthy male volunteers [13]. The effect of a morning injection of galanin was tested versus placebo in a sample of patients with depression who were on a stable treatment with trimipramine. In contrast to most other antidepressants trimipramine does not suppress REM sleep. After galanin REM latency increased and the score of the Hamilton depression rating scale decreased between morning and noon [36]. These findings point to an acute antidepressive effect of galanin.

Taken together these data can be integrated in the following model (see $\bullet$ Fig. 1). At least in men a reciprocal interaction of GHRH and CRH appears to play a key role in sleep endocrine regulation. In young healthy subjects GHRH is thought to be active during the first half of the night, resulting in the preponderance of SWS and GH and the nadirs of ACTH and cortisol. Ghrelin and galanin may act as cofactors to GHRH. Alternatively there may be a cascade of actions of these 3 peptides. Timing of sleep onset appears to be the major effect of NPY. The second half of the night is dominated by CRH possibly with somatostatin as its cofactor. During this interval REM sleep, ACTH and cortisol dominate, SWS is rare and GH secretion is low. The GHRH/ $\mathrm{CRH}$ ratio is changed in favour of $\mathrm{CRH}$ during an acute episode of depression related to $\mathrm{CRH}$ overdrive and during ageing due to reduced activity of GHRH. This may explain the similar changes of sleep endocrine activity during ageing and during depression. 


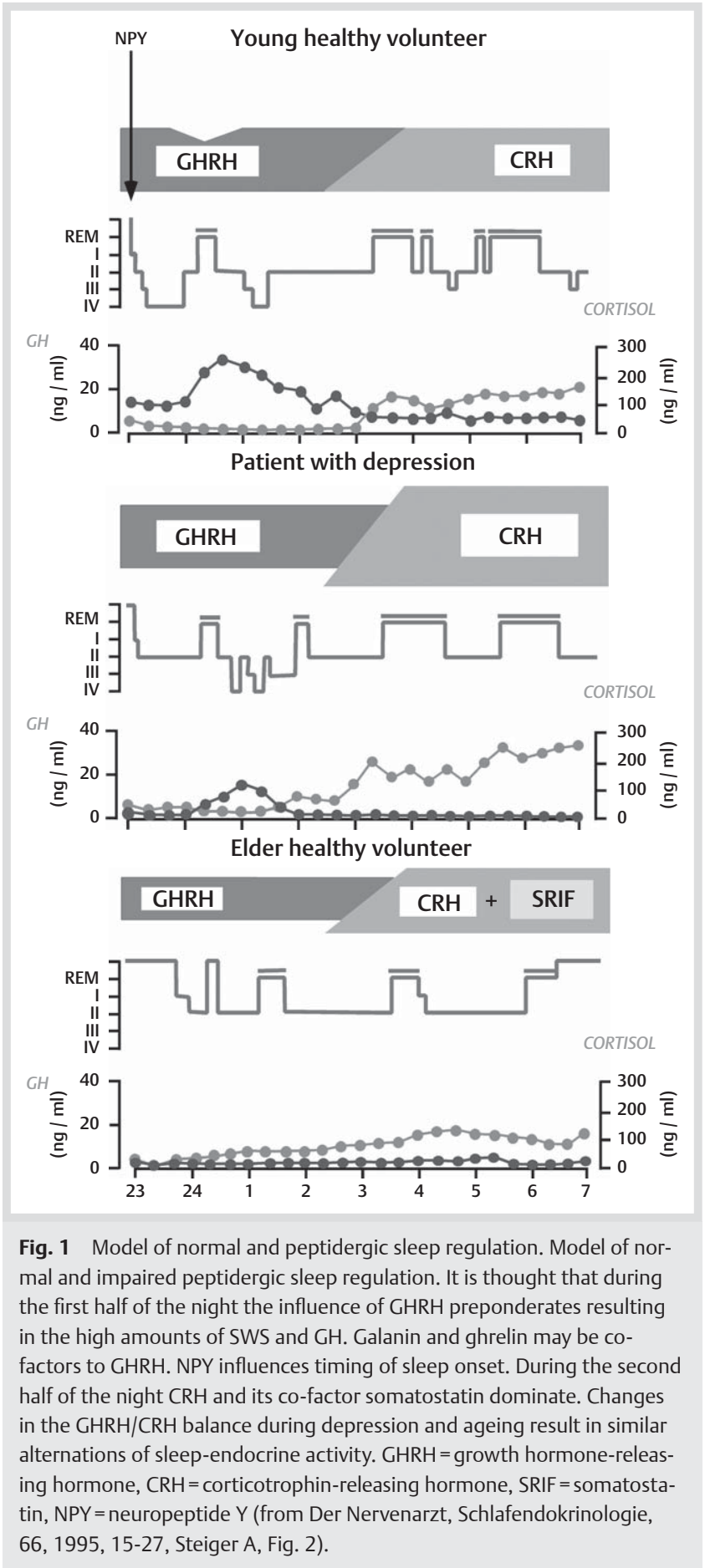

After exogenous GHRH sleep was impaired. Further studies are needed to clarify possible gender differences in sleep regulation.

\section{Sleep-Related Memory Consolidation in Depression $\nabla$}

A rapidly growing body of evidence supports a major role of sleep in the consolidation of both declarative memory and procedural skills. Furthermore, memory processes are influenced by stress hormones in a distinctive pattern. Since disturbed sleep is a major symptom and endocrine changes are a major biomarker of affective disorders, it was suggested that sleep-related neuroplasticity might be impaired in depression [37]. While cognitive impairments are indeed a well-known phenomenon in psychiatric patients, most studies of memory processes have concentrated on short-term retention periods only, demonstrating impairments specifically for the declarative memory system. When assessed before and after a night of sleep, however, the consolidation also of procedural motor skills was observed to be impaired in depressed patients: While patients treated with antidepressants and healthy control subjects showed a comparable training effect during daytime, only controls experienced further improvements after a night of sleep, while patients experienced a decrease in motor memory performance $[37,38]$. These impairments were strongly modulated by age, being most pronounced in patients above the age of 30 years and thereby coinciding with the age threshold above which depressionrelated sleep disturbances are most pronounced. Since sleeprelated memory consolidation also negatively correlated with age in healthy control subjects, this pattern of memory impairment mirrors synergistic effects of age and depression on nocturnal activity of the HPA system. Remitted patients receiving maintenance therapy with antidepressants sleep-related memory consolidation was found similar to healthy volunteers [37]. Of note, pathological alterations of HPA hormone activity diminish in remitted patients, while sleep disturbances can be observed even after remission [39].

Surprisingly, while acutely depressed patients showed worse declarative learning during the day, the sleep-related aspects of declarative memory turned out to be preserved, rendering this a double dissociation of declarative and procedural wake- and sleep-related memory impairments in depression [40]. It has been proposed that procedural memory consolidation specifically depends on REM sleep, while declarative memory consolidation depends on SWS [41]. While many antidepressants suppress REM sleep, it is unlikely that pharmacological REM sleep suppression explains overnight impairments of procedural memory in depressed patients. On the one hand, remitted patients receiving these drugs improve overnight in their procedural memory performance [37]. Furthermore, patients receiving drugs which do not suppress REM sleep like trimipramine, mirtazapine and bupropione experience sleep-related memory impairments to the same or even greater degree $[37,40]$. Likewise, healthy subjects deprived of REM sleep either pharmacologically [42] or manually [43] do not experience procedural memory impairment overnight, and a daytime nap without REM sleep may lead to procedural memory enhancement compared to a wake control condition [44]. Hence, depression- or pharmacotherapy-related alterations of sleep architecture do not seem to underlie overnight impairments of procedural memory consolidation in depressed patients. To test the hypothesis that alternatively endocrine changes play an important role in memory consolidation impairments, in a further study 4 groups we compared: i) patients with multiple sclerosis receiving glucocorticoid therapy, ii) patients with multiple sclerosis receiving mitoxantrone, iii) patients with depression and iv) healthy control subjects. Patients with multiple sclerosis after mitoxantrone and healthy subjects showed an unimpaired sleep-related memory consolidation. In contrast after glucocorticoid therapy in multiple sclerosis and in depressed patients whose cortisol levels are expected to be elevated decreases of procedural memory performance were observed after a night of sleep. These findings suggest that the lack of sleep-related memory consolidation in patients receiving exogenous gluco- 
corticoids and in depressed patients is due to elevated nocturnal HPA hormones rather than sleep-EEG changes [45].

\section{Conclusions}

Ehlers and Kupfer submitted previously [8] the "extended" 2 -process model of sleep in depression. Originally Borbély [46] suggested that a sleep-dependent process $S$ and a sleepindependent circadian process $C$ participate in sleep regulation. Furthermore, it was hypothesized that a deficiency in process $S$ accumulation plays a major role in sleep-EEG changes in depression [47]. According to Ehlers and Kupfer's model [8] GHRH and $\mathrm{CRH}$ are suggested to represent process $\mathrm{S}$ and process $\mathrm{C}$, respectively. The findings reported here support this view at least in male subjects. A change of the $\mathrm{GHRH} / \mathrm{CRH}$ ratio in favour of $\mathrm{CRH}$ appears to contribute to the characteristic changes of sleep endocrine activity in patients with depression. The resulting elevation of cortisol levels appears to contribute to the impaired memory consolidation during sleep in depression. In addition to GHRH and CRH the sleep promoting peptides galanin and ghrelin, the sleep impairing somatostatin and NPY, which influences the time of sleep onset appear to be involved in sleep regulation. Their interaction with GHRH and CRH needs further elucidation. Furthermore clarification is necessary about the role of GHRH and ghrelin in the sleep regulation of women.

\section{Acknowledgements}

$\nabla$

Supported by the Deutsche Forschungsgemeinschaft (Ste 486/5-1 to 5-4).

\section{Conflict of Interest}

$\nabla$

The authors declare no conflict of interest.

\section{References}

1 Steiger A. Neurochemical regulation of sleep. J Psychiatr Res 2007; 41: 537-552

2 Kupfer DJ. Sleep research in depressive illness: clinical implications - a tasting menu. Biol Psychiatry 1995; 38: 391-403

3 Bliwise DL. Sleep in normal aging and dementia. Sleep 1993; 16: 40-81

4 Ehlers CL, Reed TK, Henriksen SJ. Effects of corticotropin-releasing factor and growth hormone-releasing factor on sleep and activity in rats. Neuroendocrinology 1986; 42: 467-474

5 Kimura $M$, Muller-Preuss $P$, Lu A et al. Conditional corticotropinreleasing hormone overexpression in the mouse forebrain enhances rapid eye movement sleep. Mol Psychiatry 2010; 15: 154-165

6 Steiger A, Guldner J, Hemmeter $U$ et al. Effects of growth hormonereleasing hormone and somatostatin on sleep EEG and nocturnal hormone secretion in male controls. Neuroendocrinology 1992; 56: 566-573

7 Holsboer F, von Bardeleben U, Steiger A. Effects of intravenous corticotropin-releasing hormone upon sleep-related growth hormone surge and sleep EEG in man. Neuroendocrinology 1988; 48: 32-38

8 Ehlers CL, Kupfer DJ. Hypothalamic peptide modulation of EEG sleep in depression: a further application of the S-process hypothesis. Biol Psychiatry 1987; 22: 513-517

9 Mendelson WB, Slater S, Gold $P$ et al. The effect of growth hormone administration on human sleep: a dose-response study. Biol Psychiatry 1980; 15: 613-618

10 Obal J, Krueger JM. GHRH and sleep. Sleep Med Rev 2004; 8: 367-377
11 Friess $E$, von Bardeleben $U$, Wiedemann $K$ et al. Effects of pulsatile cortisol infusion on sleep-EEG and nocturnal growth hormone release in healthy men. J Sleep Res 1994; 3: 73-79

12 Steiger A, Guldner J, Knisatschek $H$ et al. Effects of an ACTH/MSH(4-9) analog (HOE 427) on the sleep EEG and nocturnal hormonal secretion in humans. Peptides 1991; 12: 1007-1010

13 Murck H, Antonijevic I, Frieboes RM et al. Galanin has REM-sleep deprivation-like effects on the sleep EEG in healthy young men. J Psychiatr Res 1999; 33: 225-232

14 Saper CB, Chou TC, Scammell TE. The sleep switch: hypothalamic control of sleep and wakefulness. Trends Neurosci 2001; 24: 726-731

15 Marshall L, Derad I, Strasburger CJ et al. A determinant factor in the efficacy of GHRH administration in promoting sleep: high peak concentration versus recurrent increasing slopes. Psychoneuroendocrinology 1999; 24: 363-370

16 Schier T, Guldner J, Colla $M$ et al. Changes in sleep-endocrine activity after growth hormone-releasing hormone depend on time of administration. J Neuroendocrinol 1997; 9: 201-205

17 Iovino M, Monteleone P, Steardo L. Repetitive growth hormone-releasing hormone administration restores the attenuated growth hormone $(\mathrm{GH})$ response to $\mathrm{GH}$-releasing hormone testing in normal aging. J Clin Endocrinol Metab 1989; 69: 910-913

18 Guldner J, Schier T, Friess $E$ et al. Reduced efficacy of growth hormonereleasing hormone in modulating sleep endocrine activity in the elderly. Neurobiol Aging 1997; 18: 491-495

19 Antonijevic IA, Murck H, Frieboes RM et al. Sexually dimorphic effects of GHRH on sleep-endocrine activity in patients with depression and normal controls - part I: the sleep EEG. Sleep Res Online 2000; 3: 5-13

20 Antonijevic IA, Murck H, Frieboes RM et al. Sexually dimorphic effects of GHRH on sleep-endocrine activity in patients with depression and normal controls - part II: hormone secretion. Sleep Res Online 2000; 3: $15-21$

21 Mathias S, Held K, Ising $M$ et al. Systemic growth hormone-releasing hormone (GHRH) impairs sleep in healthy young women. Psychoneuroendocrinology 2007; 32: 1021-1027

22 Frieboes RM, Murck H, Schier T et al. Somatostatin impairs sleep in elderly human subjects. Neuropsychopharmacology 1997; 16: 339-345

23 Ziegenbein $M$, Held K, Kuenzel HE et al. The somatostatin analogue octreotide impairs sleep and decreases EEG sigma power in young male subjects. Neuropsychopharmacology 2004; 29: 146-151

24 Schüssler $P$, Kluge $M$, Dresler $M$ et al. Sleep-impairing effect of intravenous corticotropin-releasing hormone on sleep EEG in young healthy women. Exp Clin Endocrinol Diabetes 2009; 117: 664

25 Held $K$, Künzel H, Ising $M$ et al. Treatment with the CRH1-receptorantagonist R121919 improves sleep-EEG in patients with depression. J Psychiatr Res 2004; 38: 129-136

26 Ehlers CL, Somes C, Lopez A et al. Electrophysiological actions of neuropeptide $\mathrm{Y}$ and its analogs: new measures for anxiolytic therapy? Neuropsychopharmacology 1997; 17: 34-43

27 Ehlers CL, Somes C, Seifritz E et al. CRF/NPY interactions: a potential role in sleep dysregulation in depression and anxiety. Depress Anxiety $1997 ; 6: 1-9$

28 Held K, Antonijevic I, Murck $H$ et al. Neuropeptide Y (NPY) shortens sleep latency but does not suppress ACTH and cortisol in depressed patients and normal controls. Psychoneuroendocrinology 2006; 31 : 100-107

29 Hillman JB, Tong J, Tschop M. Ghrelin biology and its role in weightrelated disorders. Discov Med 2011; 11: 521-528

30 Weikel JC, Wichniak A, Ising $M$ et al. Ghrelin promotes slow-wave sleep in humans. Am J Physiol - Endocrinol Metab 2003; 284: E407-E415

31 Kluge M, Schüssler P, Zuber $V$ et al. Ghrelin administered in the early morning increases secretion of cortisol and growth hormone without affecting sleep. Psychoneuroendocrinology 2007; 32: 287-292

32 Kluge M, Gazea M, Schüssler P et al. Ghrelin increases slow wave sleep and stage 2 sleep and decreases stage 1 sleep and REM sleep in elderly men but does not affect sleep in elderly women. Psychoneuroendocrinology 2010; 35: 297-304

33 Kluge M, Schüssler $P$, Zuber $V$ et al. Ghrelin enhances the nocturnal secretion of cortisol and growth hormone in young females without influencing sleep. Psychoneuroendocrinology 2007; 32: 1079-1085

34 Kluge $M$, Schüssler $P$, Dresler $M$ et al. Effects of ghrelin on psychopathology, sleep and secretion of cortisol and growth hormone in patients with major depression. J Psychiatr Res 2011; 45: 421-426

35 Kluge $M$, Schüssler $P$, Bleninger $P$ et al. Ghrelin alone or co-administered with GHRH or CRH increases non-REM sleep and decreases REM sleep in young males. Psychoneuroendocrinology 2008; 33: 497-506 
36 Murck H, Held K, Ziegenbein $M$ et al. Intravenous administration of the neuropeptide galanin has fast antidepressant efficacy and affects the sleep EEG. Psychoneuroendocrinology 2004; 29: 1205-1211

37 Dresler $M$, Kluge $M$, Genzel $L$ et al. Impaired off-line memory consolidation in depression. Eur Neuropsychopharmacol 2010; 20: 553-561

38 Genzel L, Ali E, Dresler $M$ et al. Sleep-dependent memory consolidation of a new task is inhibited in psychiatric patients. J Psychiatr Res 2011; 45: 555-560

39 Steiger $A$, von Bardeleben $U$, Herth $T$ et al. Sleep EEG and nocturnal secretion of cortisol and growth hormone in male patients with endogenous depression before treatment and after recovery. J Affect Disord 1989; 16: 189-195

40 Dresler $M$, Kluge $M$, Pawlowski $M$ et al. A double dissociation of memory impairments in major depression. J Psychiatr Res 2011; 45: 1593-1599

41 Plihal W, Born J. Effects of early and late nocturnal sleep on declarative and procedural memory. J Cogn Neurosci 1997; 9: 534-547
42 Rasch B, Pommer J, Diekelmann S et al. Pharmacological REM sleep suppression paradoxically improves rather than impairs skill memory. Nat Neurosci 2009; 12: 396-397

43 Genzel L, Dresler $M$, Wehrle $R$ et al. Slow wave sleep and REM sleep awakenings do not affect sleep dependent memory consolidation. Sleep 2009; 32: 302-310

44 Genzel L, Kiefer T, Renner $L$ et al. Sex and modulatory menstrual cycle effects on sleep related memory consolidation. Psychoneuroendocrinology 2012; 37: 987-998

45 Dresler M, Genzel L, Kluge $M$ et al. Off-line memory consolidation impairments in multiple sclerosis patients receiving high-dose corticosteroid treatment mirror consolidation impairments in depression. Psychoneuroendocrinology 2010; 35: 1194-1202

46 Borbély AA. A two-process model of sleep regulation. Hum Neurobiol 1982; 1: 195-204

47 Borbély AA, Wirz-Justice A. Sleep, sleep deprivation and depression: A hypothesis derived from a model of sleep regulation. Hum Neurobiol 1982; 1 : 205-210 